\title{
("ting
}

\section{DICIONÁRIO MULTILÍNGUE DO MEIO AMBIENTE: TERMOS EM PORTUGUÊS BRASILEIRO}

\author{
Flávia Cristina Cruz Lamberti ARRAES ${ }^{1}$ \\ Universidade de Brasília - UnB - DF \\ (flavialamberti@gmail.com)
}

\begin{abstract}
Resumo: No presente artigo, apresentamos uma pesquisa conduzida para a elaboração do dicionário multilíngue do meio ambiente, com termos em português brasileiro, em colaboração com o Dictionnaire fondamental de l'environnment (DiCoEnviro) desenvolvido pelo Observatoire de Linguistique SensTexte (OLST) da Université de Montréal, no Canadá. A partir de modelos léxico-semânticos aplicados à terminologia, com base em L'Homme $(2004,2015,2016)$, e a semântica de frames com base no projeto FrameNet (2015), a pesquisa foi conduzida, e está em andamento, com a finalidade de selecionar termos e identificar as suas relações terminológicas, assim como de descrever as propriedades linguísticas dos termos e conectar tal descrição a uma representação conceitual a partir da delimitação de frames.
\end{abstract}

Palavras-chave: terminologia; semântica lexical; semântica de frames; meio ambiente.

Abstract: This article presents a research carried out to prepare a multilingual dictionary on the environment with terms in Brazilian Portuguese. This project works in collaboration with the Dictionnaire fondamental de l'environnment (DiCoEnviro) developped by the Observatoire de Linguistique SensTexte (OLST) at the Université de Montréal in Canada. Based on lexical semantic approaches applied to Terminology drawing on L'Homme $(2004,2015,2016)$ and frame semantics, mainly the FrameNet project (2015), the research has been conducted with the purpose of selecting terms and identifying their terminological relationships at the same time that it describes the linguistic properties of terms and connects this description to conceptual representations based on frames.

Keywords: terminology; lexical semantics; frame semantics; environment.

Resumen: En este artículo, presentamos un estudio llevado a cabo para la preparación de diccionario multilingüe del medio ambiente, con términos en portugués brasileño, en colaboración con el Dictionnaire fondamental de l'environnement (DiCoEnviro) desarrollado por el Observatoire de Linguistique Sens-Texte (OLST) de la Université de Montréal, en Canadá. A partir de modelos léxicosemánticos aplicados a la terminología, con base en L'Homme (2004, 2015, 2016), y la semántica de frames con base en el proyecto FrameNet (2015), la investigación fue conducida, y está en marcha, con la finalidad de seleccionar términos e identificar sus relaciones terminológicas, así como de describir las propiedades lingüísticas de los términos y conectar dicha descripción a una representación conceptual a partir de la delimitación de frames.

Palabras clave: terminologia; semántica lexical; semántica de frames; medio ambiente.

\section{INTRODUÇÃO}

O Dicionário multilíngue do meio ambiente: termos em português brasileiro é um projeto de pesquisa para a descrição do léxico especializado do meio ambiente,

1 Doutora em Linguística pela UnB (2006). Professora associada do Departamento de Línguas Estrangeiras e Tradução, da área de tradução inglês-português, da UnB. 


\section{("Wîtrer}

realizado na Universidade de Brasília (UnB), no Departamento de Línguas Estrangeiras e Tradução, em colaboração com o projeto central Dictionnaire fondamental de l'environnement (Dicionário fundamental do meio ambiente), doravante DiCoEnviro, que é um recurso terminológico multilíngue, coordenado por Marie-Claude L'Homme, do Observatoire de Linguistique Sens Texte (OLST), do Département de Linguistique et Traduction (Departamento de linguística e tradução), da Universidade de Montreal (Université de Montréal), no Canadá. Neste artigo, objetivamos apresentar o quadro teórico que fundamenta a descrição dos dados no DiCoEnviro, com ênfase no português brasileiro.

O DiCoEnviro é um dicionário eletrônico que contém termos fundamentais da temática do meio ambiente em cinco línguas: francês, inglês, espanhol, italiano e português. A temática de trabalho é o meio ambiente em conjunto com outras temáticas, consideradas subdomínios: mudança climática, desmatamento, espécies em extinção, gerenciamento de resíduos e energia renovável. Cada corpus temático tem entre 500.000 a 1.200 .000 de palavras. O nível de especialização dos textos e o seu gênero textual variam, desde textos de maior grau de especialidade, como relatório, dissertações de mestrado, teses de doutorado, a textos de divulgação da informação, tais como textos jornalístico, de menor grau de especialização em relação aos primeiros.

Em português brasileiro, o DiCoEnviro já dispõe de um corpus referente a textos sobre o meio ambiente, cuja temática principal é o desmatamento, construído por Mariana Giacomini Botta, pesquisadora brasileira que realizou estágio pósdoutoral no OLST, no período de 2012-2013. A proposta de nosso projeto na UnB é dar continuidade à pesquisa nessa temática do desmatamento e ampliar o presente corpus a partir de base de dados científicas, tais como o SciELO, Portal de Periódicos da CAPES, Banco de Teses da CAPES, e de fontes de divulgação da informação, como textos jornalísticos.

O DiCoEnviro foi concebido com o propósito de ser um recurso terminológico com maior espaço para descrições de natureza linguística, uma vez que, como afirma L'Homme (2016), bancos de dados, tesauros, ontologias deixam pouco espaço para essas descrições. Além disso, "recursos que descrevem a língua 


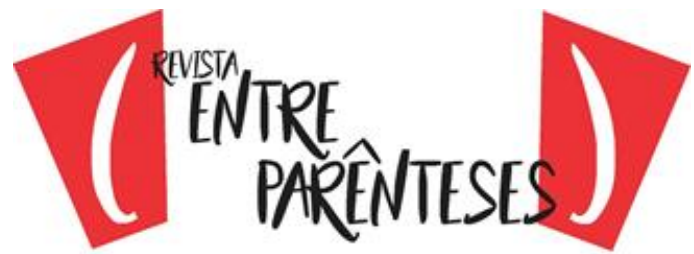

geral não têm o objetivo de dar um retrato da maneira pela qual os conhecimentos são organizados nas áreas de especialidade"2.

Nesse sentido, o modelo de descrição no DiCoEnviro busca, com base em L'Homme (2016), “...dar conta das propriedades léxico-semânticas dos termos (a estrutura argumental, o lugar do termo na rede lexical de uma língua, etc.) e realizar a conexão dessa descrição a uma representação conceitual”3.

Para desenvolver o DiCoEnviro, a pesquisa terminológica tem por base teórico-metodológica:

i) a terminologia, sob a perspectiva léxico-semântica desenvolvida em L'Homme (2004), com uso de funções lexicais para codificar as relações terminológicas (MELČUK et al. 1995, POLGUĖRE 2016);

ii) a semântica de frames, com o intuito de descrever as propriedades léxico-semânticas de unidades lexicais predicativas (L'HOMME 2012, 2015, 2016), a partir da anotação dos contextos de ocorrência.

Apresentamos a seguir os modelos teóricos adotados para a descrição do léxico do meio ambiente, mais especificamente a abordagem léxico-semântica da terminologia e a semântica de frames, com referência a dados obtidos a partir da pesquisa que retratam termos e suas relações terminológicas assim como uma amostra do frame evocado por desmatamento, desmatar e desflorestamento.

\section{DELIMITAÇÃO DO QUADRO TEÓRICO}

\subsection{A abordagem léxico-semântica da terminologia}

Na perspectiva léxico-semântica, a descrição do termo enfatiza o ambiente linguístico em que ele é usado. Nessa abordagem, o termo é considerado uma

\footnotetext{
${ }^{2}$ Texto original: De plus, les ressources générales n'ont pas comme objectif de donner un portrait de la manière dont les connaissances sont organisées dans les domaines de spécialité. (L'HOMME, 2016)

3 Texto original: (...) nous proposons un modèle de description des termes qui tient compte de leurs propriétés lexico-sémantiques (leur structure argumentale, leur place dans le réseau lexical d'une langue, etc.) et qui relie cette description à une représentation conceptuelle. (L'HOMME, 2016)
} 


\section{("titrer}

unidade lexical, cujo significado especializado é circunscrito a partir das relações paradigmáticas e sintagmáticas estabelecidas entre ele e outras unidades lexicais em uma linguagem de especialidade. Essa unidade, por estar circunscrita nessa linguagem e tecer relações com as demais unidades lexicais, tem um significado especializado justamente em razão do estabelecimento desse sistema. Nesse sentido, o objetivo da pesquisa terminológica é identificar termos e estruturas terminológicas de uma linguagem de especialidade.

As estruturas terminológicas representam evidências das relações estabelecidas entre os termos, mais precisamente das relações léxico-semânticas. Essas relações se referem ao significado diretamente, diferentemente de se concentrar na identificação de uma articulação de representações conceituais, tal como enfatizado pela abordagem tradicional da terminologia.

De acordo com L'Homme (2004, p.83), consideramos que o conjunto de termos de uma área especializada é estruturado e, nesse sentido, não são unidades linguísticas dispostas sem qualquer organização ou ligação entre si. Reforça essa consideração a afirmação de Polguère (2016, p.130):

O léxico não é um conjunto "plano", uma simples lista de lexias. Cada lexia adquire seu valor na língua a partir das múltiplas relações de oposição, de semelhança, de compatibilidade e de incompatibilidade, etc. que as conectam às outras lexias. (...) O léxico é assim uma vasta rede lexical: um sistema extremamente rico e complexo de unidades lexicais conectadas entre si. (POLGUĖRE, 2016, p.130, tradução nossa $)^{4}$

Esse sistema foi claramente apontado por Ferdinand de Saussure, no Cours de linguistique générale, conforme Polguère, (2016, p.130):

\footnotetext{
${ }^{4}$ Texto original: Le lexique n'est pas un ensemble <plat>, une simple liste de lexies. Chaque lexie prend sa valeur en langue du fait des multiples relations d'opposition, de similarité, de compatibilité, d'incompatibilité, etc., qui l'unissent aux autres lexies. (...) Le lexique est ainsi un vaste réseau lexical: un système extrêmement riche et complexe d'unités lexicales connectées les unes aux autres. (POLGUĖRE, 2016, p.130)
} 


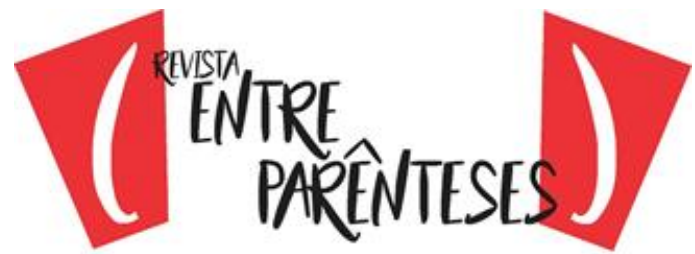

1) As relações paradigmáticas conectam as lexias no léxico por meio de conexões semânticas, possivelmente acompanhadas de conexões morfológicas.

2) As relações sintagmáticas conectam as lexias na sintaxe haja vista as afinidades combinatórias.

Nessa perspectiva, L'Homme (2004, p.90) se refere a dois tipos: i) as relações léxico-semânticas clássicas e ii) outras relações léxico-semânticas. As primeiras são paradigmáticas (e.g. hiperonímia, hiponímia, meronímia, sinonímia, antonímia), as segundas, sintagmáticas (e.g. relações actanciais e circunstanciais, combinações recorrentes ou colocações). (L'HOMME, 2004, p. 90-114)

No DiCoEnviro, as relações terminológicas são codificadas manualmente com uso de funções lexicais (Melčuk et al. 1995), tal como apresentadas na tabela 1 a seguir:

\begin{tabular}{|c|c|c|}
\hline Relação & Exemplo & Função lexical \\
\hline \multicolumn{3}{|c|}{ Significados relacionados } \\
\hline quase sinônimo & conservação $\rightarrow$ preservação & Qsyn \\
\hline sentido vizinho & conservação $\rightarrow$ proteção & Gener \\
\hline \multicolumn{3}{|l|}{ Opostos } \\
\hline Reversivo & desflorestamento $\rightarrow$ reflorestamento & $\operatorname{Rev}_{1}$ \\
\hline \multicolumn{3}{|l|}{$\begin{array}{l}\text { Outras partes do } \\
\text { discurso e derivados }\end{array}$} \\
\hline mesmo significado & desflorestamento $\rightarrow$ desflorestar & $\mathrm{S}_{0}, \mathrm{~V}_{0}$ \\
\hline $\begin{array}{l}\text { adjetivo com significado } \\
\text { adicional }\end{array}$ & sustentabilidade $\rightarrow$ sustentável & Able $_{1}$ \\
\hline \multicolumn{3}{|c|}{ Realizações linguísticas dos argumentos } \\
\hline $\begin{array}{l}\text { papel semântico (e.g. } \\
\text { agente, paciente) }\end{array}$ & $\begin{array}{l}\text { conservação de } \rightarrow \text { (paciente) } \\
\text { biodiversidade, espécie, fauna, } \\
\text { floresta }\end{array}$ & $\begin{array}{l}\text { Codificado com o nome } \\
\text { do papel semântico }\end{array}$ \\
\hline \multicolumn{3}{|l|}{ Tipos de } \\
\hline $\begin{array}{l}\text { Que é próprio de um } \\
\text { lugar }\end{array}$ & florestal $\rightarrow$ biomassa $~$ & Hypo - Lieu \\
\hline \multicolumn{3}{|l|}{ Combinações } \\
\hline $\begin{array}{l}\text { alguém ou algo tenta } \\
\text { fazer com que uma } \\
\text { ameaça não continue }\end{array}$ & ameaça $\rightarrow$ combater uma & Caus@PredMinus \\
\hline
\end{tabular}




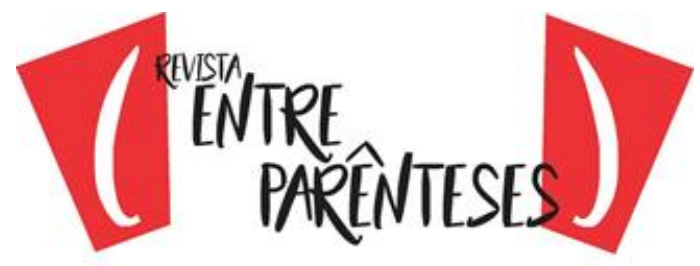

\begin{tabular}{|l|l|l|}
\hline $\begin{array}{l}\text { A extinção produz uma } \\
\text { ameaça }\end{array}$ & ameaça $\rightarrow$ causar uma & Oper $_{1}$ \\
\hline
\end{tabular}

Tabela 1: Exemplos de relações terminológicas no DiCoEnviro

A Tabela 1 apresenta uma amostra da disposição dos dados no DiCoEnviro. Nessa tabela tem-se exemplos de relações paradigmáticas, tais como Significados relacionados, Opostos, Outras partes do discurso e derivados, assim como de relações sintagmáticas com as categorias Tipos de e Combinações. Apresentam-se exemplos e a denominação da função lexical como meio de codificação da descrição proposta. Segundo L'Homme (2018), "tecnicamente a função lexical aplica-se a uma palavra-chave e gera um valor ou uma lista de valores"5. Assim, uma função lexical descreve uma relação entre uma lexia $L$ e um conjunto de lexias ou sintagmas, chamados de valores. (POLGUÈRE, 2016, p.196). Servem de exemplo as relações de quase sinonímia entre conservação e preservação, e de opostos entre desflorestamento e reflorestamento.

$\mathrm{Na}$ coluna 'relações', são fornecidas explicações do significado da função lexical, em razão da dificuldade de decifrá-las, tais como Oper ${ }_{1}$, que associa a uma lexia predicativa nominal L, no caso ameaça, o verbo suporte 'causar', cujo sujeito é o primeiro argumento de L (e.g. extinção) e L é um complemento de objeto, tal como explica Polguère (2016, p.206) a respeito da função sintagmática Oper1. Em nosso exemplo, a explicação "A extinção produz uma ameaça" indica justamente essa relação, em que extinção é o primeiro argumento da lexia predicativa 'ameaça', como em 'ameaça de extinção', e ameaça é objeto direto do verbo causar.

\subsection{Semântica de frames}

Considerando a importância de desenvolver um modelo que:

i) ressalte as propriedades linguísticas dos termos, em especial daqueles de natureza predicativa (e.g. verbos, tais como desmatar, desflorestar,

5 Texto original: Technically, an LF applies to a key word and yields one or a short list of values. (L'HOMME, 2018) 


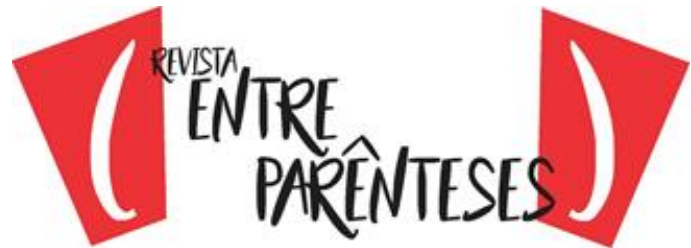

recompor, regenerar, que são base das respectivas nominalizações desmatamento, desflorestamento, recomposição, regeneração), e que

ii) permita conectar a descrição dessas propriedades ao conhecimento especializado.

O DiCoEnviro recorreu ao quadro teórico da semântica de frames (FILLMORE, 1976; FILLMORE,1982; FILLMORE; BAKER, 2010) e ao recurso FrameNet (2015). Conforme afirma L'Homme (2016), a semântica de frames considera que o léxico é construído a partir de "conhecimentos profundos" (background knowledge). A estrutura desse conhecimento é representada por meio de 'frames' definidos como cenários conceituais que reúnem as realizações no léxico"6.

Esse modelo permite:

(...) uma conexão entre as descrições linguísticas (a partir de um corpus) e um nível de representação mais abstrato, o do frame, passível de apresentar uma modelização conceitual de uma situação evocada pelo conjunto de unidades lexicais. Para a terminologia, o modelo permite lançar a hipótese de que uma ligação pode ser estabelecida entre as representações conceituais, caras à terminologia, e as descrições fundamentadas na linguística, ainda insuficientes na área. (L'HOMME, 2015, 30, tradução nossa) ${ }^{7}$

Os frames são investigados a partir da descrição linguística dos termos. A identificação de frames, definidos como uma modelização conceitual de uma situação evocada por um conjunto de unidades lexicais, permite fazer uma conexão entre a descrição linguística e o nível de representação mais abstrato. Permite, nesse sentido, realizar uma modelização conceitual, que é prerrogativa de trabalhos de terminologia, expressa por termos que evocam o frame em questão.

\footnotetext{
6 Texto original: La Sémantique des cadres (Fillmore 1976; Fillmore and Baker 2010) tient pour acquis que le lexique est construit sur la base de " connaissances d'arrière-plan " (de background knowledge). La structure de ces connaissances est représentée au moyen de "cadres " (en anglais, frames), définis comme des scénarios conceptuels qui fédèrent les réalisations dans le lexique. (L'HOMME, 2016)

7 Texto original: (...) dont celui de rendre possible une connexion entre des descriptions linguistiques (basées sur un corpus) et um niveau de représentation plus abstrait, celui du cadre, se voulant une modélisation conceptuelle d'une situation évoquée par des ensembles d'unités lexicales. Pour la terminologie, le modele permettrait de mettre en avant l'hypothèse selon laquelle um lien peut être établi entre les représentations conceptuelles chères à la terminologie et les descriptions plus ancrées dans la linguistique qui font encore cruellement défaut dans le domaine. (L'HOMME, 2015)
} 


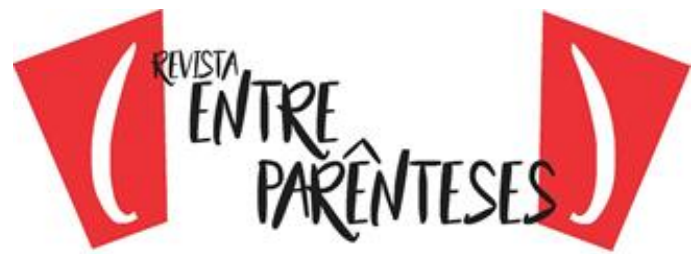

O recurso FrameNET (2015), ao indicar diversos frames, propõe i) entradas às unidades lexicais que evocam um frame e ii) anotações contextuais que se concentram na descoberta das realizações linguísticas, quer dizer, dos participantes obrigatórios (e.g. agente, causa, paciente) e dos participantes opcionais (e.g. beneficiários, circunstâncias, grau, etc.) do cenário evocado pela unidade lexical.

No caso por exemplo do meio ambiente, os termos evocam situações específicas do domínio, tais como "diferentes tipos de mudança, consequências dessas mudanças, atividades humanas que exercem impacto no meio ambiente, medidas tomadas para impedir a degradação, etc.", indicadas por L'Homme (2016). A descoberta dessas realizações linguísticas permite conhecer os participantes obrigatórios e opcionais que constituem o cenário do frame, que são analisados por meio da anotação dos contextos. Serve como exemplo, a seguir, os contextos de ocorrência que denotam o processo segundo o qual árvores são removidas de um local:

1) De acordo com a meta anual de produção, a empresa DESMATA, atualmente, áreas que variam de 400 a 500 ha por ano.

2) Os objetivos geopolíticos...complementaram os objetivos econômicos estritamente privados da ocupação e DESMATAMENTO da região.

3) O Banco Mundial vem, há décadas, dialogando com o governo brasileiro sobre projetos (...). Dentre as questões fundamentais deste diálogo está o entendimento sobre o processo de DESFLORESTAMENTO da região.

Os termos denotam um argumento em comum, o fato de se referirem à origem do processo: região, áreas, e foram reunidos em um frame denominado 'Removing trees from location' (removendo árvores de um local), ilustrado a seguir:

Removing_trees_from_location

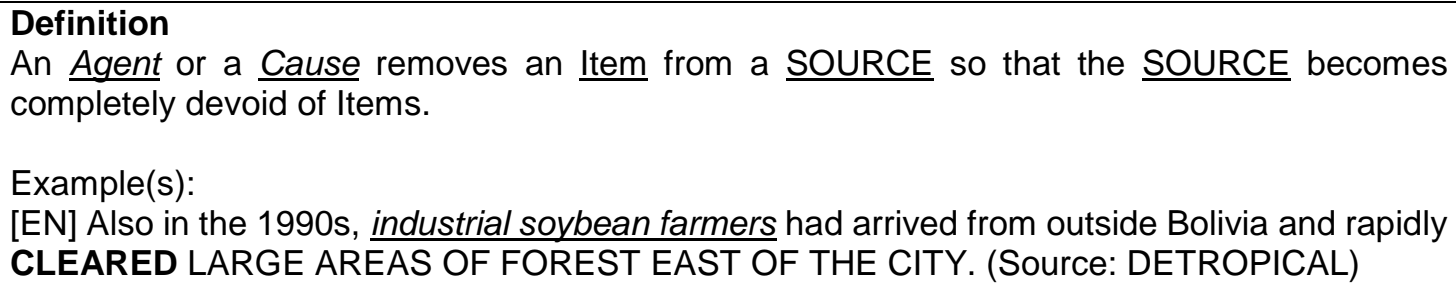


[FR] En effet, le DÉFRICHEMENT de la végétation acentue l'érosion qui se traduit par la perte d'énormes quantités de matières solides arrachées aux sols et transportées par ruissellement jusqu'au cours d'eau. (Source: FRAPROB)

[POR] Mesmo a questão estando na justiça, o Dnocs DESMATOU PARTE SIGNIFICATIVA DO TERRITÓRIO ocupado pelos Tremembé de Queimadas, principalmente os "serrotes", (...) (Source: MANEJOAGRICOLA_2012)

Notes: This frame was defined for the DiCoEnviro.

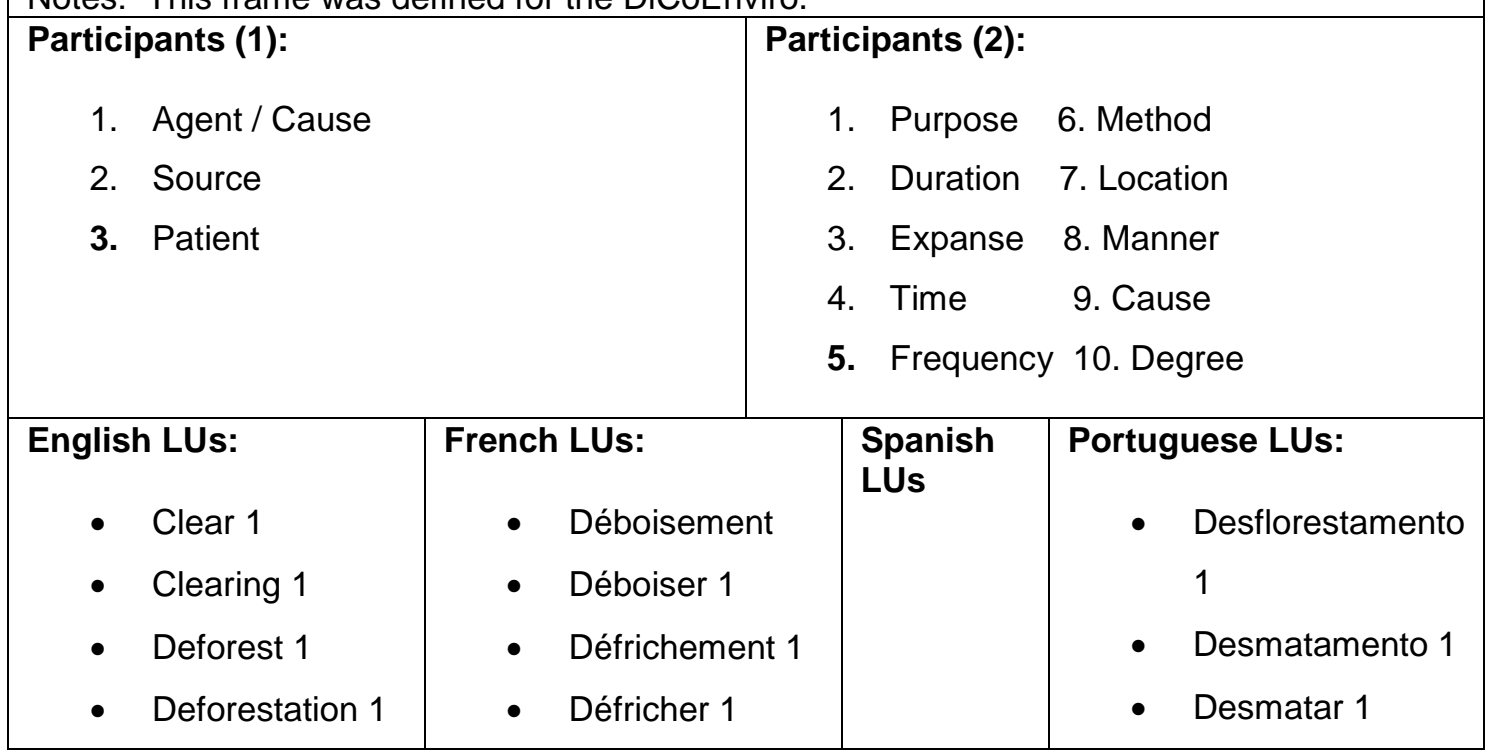

Tabela 2: Frame "Removing trees from location". Fonte http://olst.ling.umontreal.ca/dicoenviro/framed/index.php

O frame acima compreende uma definição em inglês, exemplos de contextos em inglês, francês e português. Logo abaixo, seguem os participantes obrigatórios (marcados pelo número 1, referindo-se aos elementos centrais do frame, no caso agente, causa, paciente) e os participantes opcionais (marcados pelo número 2, que se referem aos elementos periféricos do frame, tais como propósito, tempo, duração e outros).

Por último, apresentam-se os termos em inglês, francês e português, com espaço previsto para o espanhol, que evocam o frame. É possível clicar em cada um deles para acessar as entradas lexicais no DiCoEnviro ${ }^{8}$.

\footnotetext{
${ }^{8}$ Ressalte-se a criação pelo OLST de um recurso recente, denominado Neovisual, que possibilita a navegação virtual, por meio de expansões gráficas tal como apresentadas em L'Homme (2018), em toda a rede terminológica descrita (a estrutura argumental, as relações terminológicas, os equivalentes). $\mathrm{O}$ acesso é disponibilizado no canto direito da entrada do verbete ao clicar na imagem 8
} 


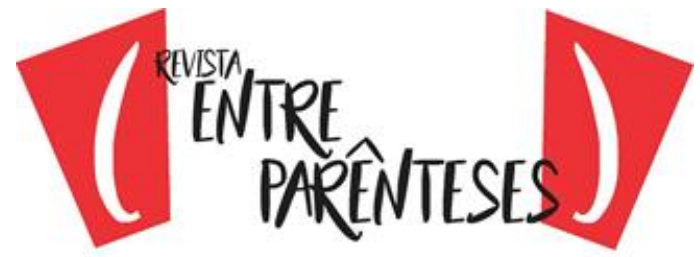

\section{CONSIDERAÇÕES FINAIS}

Este trabalho apresentou projeto de pesquisa para a descrição linguística do léxico especializado referente ao meio ambiente em português brasileiro, realizado em colaboração com o Observatoire de Linguistique Sens-Texte (OLST) do Département de Linguistique et traduction da Universidade de Montreal no Canadá. A terminologia, com foco na abordagem léxico-semântica, e a semântica de frame compõem o quadro teórico e metodológico para a realização da pesquisa.

Como resultado, tem-se a preparação, contínua e constante, de recursos terminológicos, mais especificamente o DiCoEnviro e o Framed DiCoEnviro (versão em frames do DiCoEnviro) que possibilita o registro de termos e das relações terminológicas possibilitando apresentar a rede lexical que constitui o léxico especializado do meio ambiente do português brasileiro. Em razão de o projeto ser multilíngue, essa rede lexical é colocada ao lado de redes terminológicas em francês, inglês, espanhol e italiano. A versão em frames do DiCoEnviro, o FramedDiCoEnviro, possibilita enfatizar as propriedades linguísticas dos termos, especial para dar conta das unidades predicativas, aquelas cuja definição depende de seus argumentos, e estabelecer a conexão dessas propriedades com uma representação conceitual a partir da delimitação de frames.

\section{REFERÊNCIAS}

DiCoEnviro. Dictionnaire fundamental de l'environnement. Disponível em: http://olst.ling.umontreal.ca/cgi-bin/dicoenviro/search.cgi. Acesso em out. 2018.

FILLMORE, C.J. Frame Semantics and the Nature of Language. In: Annals New York Academy of Sciences: Conference on the Origin and Development of Language and Speech, n. 280, 1976. p. 20-32.

Frame Semantics. In: Linguistics in the Morning Calm, Seoul: Hanshin Publishing Co., 1982. p. 111-137.

FILLMORE, C.J.; BAKER, C. A Frames Approach to Semantic Analysis. In: HEINE, B.; NARROG, H. (org.). The Oxford Handbook of Linguistic Analysis. Oxford: Oxford University Press, 2010. p. 313-339. 
FrameNet. Disponível em: https://framenet.icsi.berkeley.edu/fndrupal/. Acesso em set 2018.

L'HOMME, M.C. La terminologie: principes et techniques. Montréal: LesPresses de l'Université de Montréal, 2004.

Adding Syntactico-semantic Information to Specialized Dictionaries: An Application of the FrameNet Methodology. In: GOUWS, R. et al. (org.). Lexicographica, 2012, n. 28. p. 233-252.

L'HOMME, M.C., ROBICHAUD, B. Frames and Terminology: Representing Predicative Units in the Field of the Environment. In: Cognitive Aspects of the Lexicon (Cogalex 2014). Dublin, Irlanda, 2014.

L'HOMME, M.C. Découverte de cadres sémantiques dans le domaine de l'environnement : le cas de l'influence objective. Terminàlia 12, p. 29-40, 2015.

L'HOMME, M.C. Terminologie de l'environnement et sémantique des cadres. SHS Web of Conferences 27 05010, Congrès Mondial de Linguistique Française, 2016.

L'HOMME, M,C, ROBICHAUD, B; PRÉVIL, N. Browsing the Terminological Structure of a Specialized Domain: A Method Based on Lexical Functions and their Classification, Language Resources and Evaluation. LREC 2018, 3079-3086, Miyazaki, Japão, 2018.

MELCUK, I.; CLAS,A.; POLGUÈRE, A. Introduction a la lexicologie explicative et combinatoire. Louvain-la-Neuve, Bélgica: Duculot, 1995.

POLGUÈRE, A. Lexicologie et sémantique lexicale. Notions fundamentales. 3ed. Montreal: Presses de L'Université de Montreal, 2016.

Recebido em 20/10/2018.

Aceito em 07/12/2018. 